\title{
ON THE UNIVERSAL COVERING SPACE AND THE FUNDAMENTAL GROUP
}

\author{
C. W. SAALFRANK
}

In this note it will be shown that when $A$ is a retract (cf. [3]) ${ }^{1}$ of $X$, then the same relationship holds between the universal covering spaces of $A$ and $X$ (Theorem 1.1) and also between the fundamental groups of $A$ and $X$ (Theorem 2.2).

We denote the universal covering space of $X$ by $X_{p}^{*}$, where $p \in X$, and the fundamental group by $\pi(X)[1]$. All spaces are assumed to be arcwise connected, Hausdorff spaces. If $\alpha$ and $\beta$ are continuous maps of the closed unit interval $(0,1)$ into $X$, then we use the notation $\alpha \sim \beta$ (equivalent) to mean:

$\alpha(0)=\beta(0), \alpha(1)=\beta(1)$, and there exists a continuous map $k(t, s)$ such that

(i) $k:(0,1) \times(0,1) \rightarrow X$ (into),

(ii) $k(t, 0)=\alpha(t), k(t, 1)=\beta(t)$ for all $t \in(0,1)$,

(iii) $k(0, s)=\alpha(0)=\beta(0), k(1, s)=\alpha(1)=\beta(1)$ for all $s \in(0,1)$.

A continuous map $k$ satisfying the above conditions $\mathrm{i}$, ii, and iii will be said to satisfy the E-conditions for $(\alpha, \beta, X)$.

1. Lemma 1.1. If $A$ is a retract of $X$ and $p \in A$, then $A_{p}^{*}$ is homeomorphic to a subset of $X_{p}^{*}$.

Proof. Consider any $a^{*} \in A_{p}^{*}$ and any way $\alpha \in a^{*}$. Then $\alpha:(0,1)$ $\rightarrow A$. Define $\alpha^{\prime}:(0,1) \rightarrow X$ by $\alpha^{\prime}(t)=\alpha(t)$ for all $t \in(0,1)$, and let $x^{*}$ be the element of $X_{p}^{*}$ such that $\alpha^{\prime} \in x^{*}$. Defining $h\left(a^{*}\right)=x^{*}$, it is easily shown that $h$ is single-valued.

To show that $h$ is continuous, we consider any neighborhood $U^{*}(U, \alpha)$ of $x^{*}$ where $h\left(a^{*}\right)=x^{*}$. Then $U^{*}(U, \alpha)$ consists of all $g^{*}$ in $X_{p}^{*}$ such that $g^{*} \supset \alpha \beta$ with $\beta(t) \subset U$ for all $t \in(0,1)$, and where $\alpha \in x^{*}$ and $U$ is any neighborhood in $X$ of $\alpha(1)$. Consider any $\gamma \in a^{*}$. Then $\gamma^{\prime} \in x^{*}$ and therefore $\gamma^{\prime} \sim \alpha$. Hence there exists a continuous map $k^{\prime}:(0,1) \times(0,1) \rightarrow X$ such that $k^{\prime}$ satisfies the E-conditions for $\left(\gamma^{\prime}, \alpha, X\right)$. We define $k:(0,1) \times(0,1) \rightarrow A$ by $k(t, s)=r k^{\prime}(t, s)$. It follows easily that $r \alpha(0)=\gamma(0), r \alpha(1)=\gamma(1)$, and that $k$ satisfies the E-conditions for $(r \alpha, \gamma, A)$. Hence $r \alpha \sim \gamma$ and we have $r \alpha \in a^{*}$. Take the neighborhood $V^{*}(U \cap A, r \alpha)$ of $a^{*}$. Now consider any $a_{1}^{*}$ $\in V^{*}(U \cap A, r \alpha)$ and let $h\left(a_{1}^{*}\right)=x_{1}^{*}$. Then $a_{1}^{*}$ contains a way $(r \alpha) \beta$

Received by the editors May 29, 1952 and, in revised form, January 12, 1953.

${ }^{1}$ Numbers in brackets refer to the bibliography at the end of the paper. 
where $\beta(t) \subset U \cap A$ for all $t \in(0,1)$ and $x_{1}^{*}$ contains the way $[(r \alpha) \beta]^{\prime}$ $=(r \alpha)^{\prime} \beta^{\prime}$. Since $r \alpha \in a^{*}$ we have $(r \alpha)^{\prime} \in x^{*}$ and hence $(r \alpha)^{\prime} \sim \alpha$. Therefore $(r \alpha)^{\prime} \beta^{\prime} \sim \alpha \beta^{\prime}$ and $x_{1}^{*} \in U^{*}(U, \alpha)$. Hence $h$ is continuous.

Using the fact that $A$ is a retract of $X$, it follows easily that the $\operatorname{map} h$ is one-to-one.

To show $h^{-1}$ is continuous, consider any $b^{*} \in h\left(A_{p}^{*}\right) \subset X_{p}^{*}$. Let $h^{-1}\left(b^{*}\right)=a^{*}$ and take any neighborhood $U^{*}(U \cap A, \alpha)$ of $a^{*}$ where $U$ is a neighborhood in $X$. Since $r$ is continuous and $r(\alpha(1))=\alpha(1)$, there exists a neighborhood $V$ of $\alpha(1)$ in $X$ such that $r(V) \subset U \cap A$. Take the neighborhood $V^{*}\left(V, \alpha^{\prime}\right) \cap h\left(A_{p}^{*}\right)$ of $b^{*}$ and consider any $b_{1}^{*} \in V^{*}\left(V, \alpha^{\prime}\right)$ $\cap h\left(A_{p}^{*}\right)$. Then $b_{1}^{*} \supset \alpha^{\prime} \beta$, where $\beta(t) \subset V$ for all $t \in(0,1)$. It follows easily that $h^{-1}\left(b_{1}^{*}\right) \supset r\left(\alpha^{\prime} \beta\right)=\alpha r(\beta)$. Since $r(\beta(t)) \subset U \cap A$ for all $t$ $\in(0,1)$, we have that $h^{-1}\left(b_{1}^{*}\right) \in U^{*}(U \cap A, \alpha)$ and hence $h^{-1}$ is continuous.

Theorem 1.1. If $A$ is a retract of $X$ and $p \in A$, then $A_{p}^{*}$ is a retract of $X_{p}^{*}$.

Proof. Let $h$ be the homeomorphism given by Lemma 1.1 and let $h\left(A_{p}^{*}\right)=B^{*} \subset X_{p}^{*}$. We shall show that $B^{*}$ is a retract of $X_{p}^{*}$. Let $r$ be the map that retracts $X$ onto $A$, and we shall consider $r$ to be a map of $X$ into $X$. Consider any $x^{*} \in X_{p}^{*}$ and any way $\alpha \in x^{*}$. Then $r \alpha:(0,1)$ $\rightarrow X$, and therefore there exists an element $b^{*} \in X_{p}^{*}$ such that $r \alpha \in b^{*}$. We define the map $R: X_{p}^{*} \rightarrow B^{*}$ by $R\left(x^{*}\right)=b^{*}$. Since $r \alpha(t) \subset A$ for all $t \in(0,1)$, clearly $b^{*} \in B^{*}$ and $R\left(X_{p}^{*}\right) \subset B^{*}$. It is easy to show that $R$ is single-valued.

For any $b^{*} \in B^{*}$, there exists a way $\alpha^{\prime} \in b^{*}$ such that $\alpha^{\prime}(t) \subset A$ for all $t \in(0,1)$. Hence $R\left(b^{*}\right)=b^{*}$.

To show $R$ is continuous, consider any $x^{*} \in X_{p}^{*}$ and let $R\left(x^{*}\right)=b^{*}$. Consider any neighberhood $U^{*}(U, \alpha) \cap B^{*}$ of $b^{*}$. For any $\gamma \in x^{*}$ we have $r \gamma \in b^{*}$. Hence $r \gamma \sim \alpha$ and $r \gamma(1)=\alpha(1)$. Since $r$ is continuous, for the neighborhood $U$ of $\alpha(1)$, there exists a neighborhood $V$ of $\gamma(1)$ such that $r(V) \subset U$. Take the neighborhood $V^{*}(V, \gamma)$ of $x^{*}$ and consider any $x_{1}^{*} \in V^{*}(V, \gamma)$. Now $x_{1}^{*} \supset \gamma \beta$ where $\beta(t) \subset V$ for all $t \in(0,1)$. There exists $b_{1}^{*}$ such that $r(\gamma \beta) \in b_{1}^{*}$ and we have $R\left(x_{1}^{*}\right)=b_{1}^{*}$. Clearly $r(\gamma \beta)=(r \gamma)(r \beta) \sim \alpha(r \beta)$, and hence we have $\alpha(r \beta) \in b_{1}{ }^{*}$. But since $r \beta(t) \subset U$ for all $t \in(0,1)$, this means that $b_{1}^{*} \in U^{*}(U, \alpha) \cap B^{*}$.

2. Definition. Let $C \in \pi(X), \alpha \in C$. Let $x^{*} \in X_{p}^{*}, \beta \in x^{*}$. Define $K_{C}\left(x^{*}\right)$ to be the element of $X_{p}^{*}$ which contains $\alpha \beta$.

It is well known that $K_{C}$ is a covering homeomorphism (i.e. $K_{C}: X_{p}^{*} \rightarrow X_{p}^{*}$ is a homeomorphism such that $L\left(x^{*}\right)=L K_{C}\left(x^{*}\right)$ for all $x^{*} \in X_{p}^{*}$ where $L$ is the natural map defined by $L\left(x^{*}\right)=\alpha(1)$, for $\left.\alpha \in x^{*}\right)$. Moreover, it is well known that the set $\left\{K_{C}\right\}$ of all such $K_{C}$ 
is simply the set of all covering homeomorphisms on $X_{p}^{*}$ and forms a group.

For the work that follows we assume $A$ is a retract of $X$. Letting $h$ be the homeomorphism given by Lemma 1.1 and letting $h\left(A_{p}^{*}\right)$ $=B^{*} C X_{p}^{*}$ it is easy to show the following result.

Theorem 2.1. Let $C \in \pi(X)$ and let $\left\{K_{C}\right\}$ be the collection of all covering homeomorphisms on $X_{p}^{*}$. Let $C_{1} \in \pi(X)$ such that $C_{1} \supset \alpha$ such that $\alpha(t) \in A$ for all $t$. Then the collection $\left\{K_{C_{1}}\right\}$ is the set of all covering homeomorphisms on $B^{*}$. That is, $\left\{K_{C_{1}}\right\}$ consists of all $K_{C} \in\left\{K_{C}\right\}$ such that $K_{C}: B^{*} \rightarrow B^{*}$ (onto).

Definition. A subgroup $F$ of a group $G$ is called a retract of $G$ provided there exists a homomorphism $h$ such that $h(G)=F$ and such that $h(f)=f$ for all $f \in F$.

It is easy to show that $\left\{K_{C_{1}}\right\}$ is a subgroup of the group $\left\{K_{C}\right\}$.

LEMMA 2.1. The subgroup $\left\{K_{C_{1}}\right\}$ is a retract of the group of all covering homeomorphisms $\left\{K_{c}\right\}$ of $X_{p}^{*}$, and $\left\{K_{C_{1}}\right\}$ is isomorphic with the group of all covering homeomorphisms $\left\{M_{C}\right\}$ of $A_{p}^{*}$.

Proof. Define a homomorphism $H:\left\{K_{C}\right\} \rightarrow\left\{K_{C_{1}}\right\}$ as follows. Consider any $K_{C} \in\left\{K_{C}\right\}$ and any $\alpha \in C$. Then considering the retraction map $r(X)=A$ as $r: X \rightarrow X$ (into), we have $r \alpha:(0,1) \rightarrow X$. There exists an element $C_{1}$ in $\pi(X)$ such that $r \alpha \in C_{1}$. Define $H\left(K_{C}\right)=K_{C_{1}}$. Clearly $K_{C_{1}} \in\left\{K_{C_{1}}\right\}$ since $r \alpha \in C_{1}$ and $r \alpha(t) \subset A$ for all $t$. It is easy to show that the homomorphism $H$ is single-valued.

For $K_{C_{1}} \in\left\{K_{C_{1}}\right\}, C_{1} \supset \alpha$ such that $\alpha(t) \subset A$ for all $t$. Hence $r \alpha(t)$ $=\alpha(t)$ for all $t$ and $H\left(K_{C_{1}}\right)=K_{C_{1}}$.

Consider any $K_{B}$ and $K_{C}$ in $\left\{K_{C}\right\}$ and consider any $\beta \in B$ and $\gamma \in C$. It follows easily that $K_{B} K_{C}=K_{B C}$ and hence we have $H\left(K_{B} K_{C}\right)=H\left(K_{B C}\right)=K_{(B C)_{1}}$ where $\beta \gamma \in B C$ and $r(\beta \gamma) \in(B C)_{1}$. Also $H\left(K_{B}\right) H\left(K_{C}\right)=K_{B_{1}} K_{C_{1}}=K_{B_{1} C_{1}}$ where $r \beta \in B_{1}, \quad r \gamma \in C_{1}$ and hence $(r \beta)(r \gamma) \in B_{1} C_{1}$. But $r(\beta \gamma)=(r \beta)(r \gamma)$ and therefore $(B C)_{1}=B_{1} C_{1}$. Hence $H\left(K_{B} K_{C}\right)=H\left(K_{B}\right) H\left(K_{C}\right)$.

Define an isomorphism $F:\left\{M_{C}\right\} \rightarrow\left\{K_{C_{1}}\right\}$ (onto) as follows. Consider any $M_{C} \in\left\{M_{C}\right\}$ and any $\alpha \in C \in \pi(A)$. Since $\alpha:(0,1) \rightarrow A$, we define $\alpha^{\prime}(t)=\alpha(t)$ for all $t$ and have $\alpha^{\prime}:(0,1) \rightarrow X$. There exists $C_{1}$ $\in \pi(X)$ such that $\alpha^{\prime} \in C_{1}$. Define $F\left(M_{C}\right)=K_{C_{1}}$.

It is well known that the fundamental group $\pi(X)$ of $X$ is isomorphic with the group $\left\{K_{c}\right\}$ of all covering homeomorphisms of $X_{p}^{*}$. Hence using Lemma 2.1 we have the result.

THEOREM 2.2. If $A$ is a retract of $X$, then the fundamental group $\pi(A)$ is a retract of the fundamental group $\pi(X)$. 


\section{BIBLIOGRAPHY}

1. H. Seifert and W. Thelfall, Lehrbuch der Topologie, Leipzig and Berlin, 1934.

2. A. M. Peiser, Covering mappings, Duke Math. J. vol. 10 (1943) pp. 305-307.

3. K. Borsuk, Sur les retractes, Fund. Math. vol. 17 (1931) pp. 152-170.

4. C. W. Saalfrank, Retraction properties for normal Hausdorff spaces, Fund. Math. vol. 36 (1949) pp. 93-108.

RUTGERS UNIVERSITY AND

LAFA YetTE COLLEGE

\section{DENSE IMBEDDING OF TOPOLOGICAL GROUPS}

\section{MORIKUNI GOTO 1}

The present article originated from the problem of determining when a continuous representation of a Lie group into another is open. Important cases of the problem have been discussed by A. Malcev² and the author, ${ }^{8}$ and some of them have been extended to the case of more general groups by the author and H. Yamabe.4

Recently W. T. van Est obtained new results concerning the same problem on Lie groups. ${ }^{5}$ Here I shall give an extension of his essential result to a more general case in a simpler way.

Let $G$ be a locally compact connected group and let $A(G)$ be the group of all continuous automorphisms of $G$. Let $A(G)$ be topologized by the notion of uniform convergence in the wider sense.

Now let $I(G)$ be the subgroup of $A(G)$ composed of all inner automorphisms of $G$. We shall call $G$ a (CA) group ${ }^{6}$ if $I(G)$ is a closed subgroup of $A(G)$.

LeмmA. ${ }^{7}$ Let $G$ be a locally compact connected and locally connected group, and $H$ a locally compact group. If $\phi$ is a continuous isomorphism which maps $G$ in an everywhere dense subgroup in $H$, then the following propositions hold:

(1) $\phi(G)$ is an invariant subgroup of $H$.

(2) Let $h$ be an element of $H$. Let us consider the automorphism $\sigma_{h}(x)$ defined by $\sigma_{h} x=\phi^{-1}\left(h^{-1} \phi(x)\right) h$ for $x \in G$. Then $\sigma_{h}(x)$ is a continuous

Received by the editors October $6,1952$.

1 The author's name will be hereafter spelled "Goto" instead of "Goto" which has been used thus far.

${ }^{2}$ Malcev [4] in the bibliography.

${ }^{3}$ Goto [1].

4 Goto and Yamabe [3]. See also [2].

${ }^{5}$ van Est [5], where he solved a prize problem (Wiskundig Genootshap Amsterdam, 1950), which had already been established in [1] and generalized in [3], independently of the author.

- The notion of a (CA) group is a generalization of van Est's (CA) Lie group.

${ }^{7}$ See $[3]$ and [2]. 